\title{
Lifetimes of the image-state resonances at metal surfaces
}

\author{
A. G. Borisov, ${ }^{1,2}$ E. V. Chulkov, ${ }^{2,3}$ and P. M. Echenique ${ }^{2,3,4}$ \\ ${ }^{1}$ Laboratoire des Collisions Atomiques et Moléculaires (CNRS UMR 8625), Bâtiment 351, Université Paris-Sud, \\ 91405 Orsay Cedex, France \\ ${ }^{2}$ Donostia International Physics Center DIPC, P. Manuel de Lardizabal 4, 20018 San Sebastián, Spain \\ ${ }^{3}$ Departamento de Física de Materiales, Facultad de Químicas UPV/EHU, Apartado 1072, 20080 San Sebastián, Spain \\ ${ }^{4}$ Unidad de Física de Materiales, Centro Mixto CSIC-UPV/EHU, P. Manuel de Lardizabal 3, 20018 San Sebastián, Spain
}

(Received 20 December 2005; published 10 February 2006)

\begin{abstract}
We apply the wave packet propagation approach to study electronic relaxation of excited states at surfaces. On the example of the image-state resonances on $\mathrm{Cu}(111), \mathrm{Ag}(111), \mathrm{Au}(111)$, and $\mathrm{Be}(0001)$ surfaces we discuss the relative role of inelastic electron-electron scattering and resonant one-electron transfer in the decay of the population of excited states. Present theoretical results are in a good agreement with available experimental data. An $n^{3}$ scaling of the resonance lifetimes with quantum number $n$ is obtained in our study. It can be understood from the semiclassical picture for the Rydberg electron motion.
\end{abstract}

DOI: $10.1103 /$ PhysRevB.73.073402

PACS number(s): 73.20.At, 72.15.Lh

Understanding of the dynamics of electronic excitations at surfaces and interfaces is of both fundamental interest and technological importance. Thus, transport of excited electrons through the interface is a key factor for molecular electronics and scanning tunneling microscopy (STM). ${ }^{1}$ Moreover, excited states are often invoked as reaction intermediates in various chemical processes at surfaces induced by photon, electron, or atom (molecule) impact. ${ }^{2}$ The time evolution of the excited states is determined by the dephasing and population decay processes. The pure dephasing process corresponds to the decay of the coherence between various states without loss of their population. The population decay governs the survival of the excited state and thus, e.g., the possibility of this state to drive the system into a given reaction channel. In this context, two fundamentally different aspects of the decay of the electronically excited states arise: (i) The states are stationary within the oneelectron picture and decay inelastically because of the coupling to the bath of many-body excitations; (ii) the states are quasistationary (resonances) within the one-electron picture and thus can decay via energy-conserving one-electron transfer. Recent development of the time-resolved twophoton-photoemission (TR2PPE) spectroscopy in the femtosecond regime allowed direct access to the time evolution of the excited states at surfaces. ${ }^{3-5}$ On the theoretical side, description of the excited-state dynamics is still a challenge. This is why it is very important to study both types of the population decay for well-defined systems, where theoretical results can be compared with TR2PPE data.

The image states (ISs) and image-state resonances (ISRs) offer such a possibility. These states arise because of the self-interaction with the polarization charge which traps an excited electron in front of the surface. Far from the surface this self-interaction converges to the classical image potential. When electron penetration into the metal is prohibited by the projected band gap, ISs are stationary within the oneelectron picture. They form a Rydberg series converging toward the vacuum level with energies given by ${ }^{6-9}$ (atomic units are used throughout the paper unless otherwise stated)

$$
E_{n}=-\frac{1}{32(n+\delta)^{2}}
$$

where $n=1,2, \ldots$ is the principal quantum number, and $\delta$ the quantum defect. The electron motion parallel to the surface is quasifree with effective mass close to 1 . Decay of electrons excited in the IS at clean surfaces proceeds via energy relaxation through inelastic electron-electron scattering as has been thoroughly studied both experimentally and theoretically. $3,4,10-13$

Strikingly much less information is available on the ISRs. In contrast to ISs, energies of the ISR lie within the energy range of the projected bulk electronic states. The ISRs can then decay by one-electron transition: the energy-conserving resonant electron transfer (RET) into the bulk metal states. The RET is a priori more efficient than multielectron scattering. This explains short (as compared to the ISs) lifetimes of the ISRs and thus difficulty in their characterization. The ISRs can be stabilized at surfaces coated with molecular spacer layers, where change in their lifetimes with layer thickness gives valuable information on the electronic transport through the interface. ${ }^{14}$ From the theoretical side, most of the works addressed the energy positions of the ISRs, leaving open the question of their lifetimes. ${ }^{15-17}$

In this Brief Report we present a theoretical study of the binding energies and, in particular, lifetimes of the imagestate resonances on clean metal surfaces. Systematic calculations performed within a wide $n$ range (1-6) for several surfaces $\mathrm{Cu}(111), \mathrm{Ag}(111), \mathrm{Au}(111)$, and $\mathrm{Be}(0001)$ allow discussion of the dependence of the ISR properties on the surface band structure. We find and explain an $n^{3}$ scaling of the ISR lifetimes with the principal quantum number $n$. Results of the present calculation are in a good agreement with available experimental data.

Our study is based on the wave packet propagation approach. Details of the method can be found elsewhere. ${ }^{18}$ In brief, the time-dependent one-electron Schrödinger equation is solved numerically on a grid of equidistant points in the $z$ coordinate chosen along the surface normal. The time evolu- 
TABLE I. Quantum defect $\delta$ for the IS resonances.

\begin{tabular}{lccc}
\hline \hline $\mathrm{Au}(111)$ & $\mathrm{Ag}(111)$ & $\mathrm{Cu}(111)$ & $\mathrm{Be}(0001)$ \\
\hline 0.01 & 0.007 & -0.026 & -0.107 \\
\hline \hline
\end{tabular}

tion of the electron wave function $\psi(z, t)$ is described with the split-operator technique:

$$
\psi(z, t+\Delta t)=e^{-i(\Delta t / 2) V} e^{-i \Delta t T} e^{-i(\Delta t / 2) V} \psi(z, t)
$$

where $T$ is the kinetic energy operator calculated within the Fourier pseudospectral approach, ${ }^{19}$ and $V$ is the local effective potential. The initial wave function $\psi(z, t=0) \equiv \psi_{0}(z)$ is taken as a superposition of several Gaussians centered in the vacuum side.

The effective potential is a function only of $z$, and it is given by

$$
V(z)=V_{s}(z)+V_{b}(z)+V_{0}(z)
$$

where $V_{s}(z)$ is the model potential for electron-metal interaction obtained on the basis of ab initio studies. ${ }^{16}$ Many-body calculations based on this potential have been previously shown to account for the experimental data on the energies and lifetimes of the ISs. ${ }^{11,12}$ In order to avoid artifacts of the finite size of the computation mesh, an absorbing potential ${ }^{20}$ $V_{b}(z)$ is introduced at the grid boundaries. Convergent results do not depend on the specific choice of $V_{b}(z)$. The effect of the many-body interactions on the lifetimes of the ISRs has been assessed with calculations where, analogous to lowenergy electron diffraction studies, an optical potential $V_{0}(z)$ has been added everywhere inside the metal. ${ }^{21}$

Description of ISRs with high $n$ required a fairly large size of the spatial mesh: $-200 \leqslant z \leqslant 500$ a.u. (positive values of $z$ stand for the vacuum side, and $z=0$ coincides with the image plane). Typically the mesh comprises 4096 knots. The propagation time step is $\Delta t=0.05$.

From $\psi(z, t)$ we calculate the projected density of states $\rho(\omega)$ given by the real part of the time-to-frequency Laplace transform of the autocorrelation function $A(t)=\left\langle\psi_{0} \mid \psi(t)\right\rangle$. Quasistationary states appear as peak structures in $\rho(\omega)$. Fitting these structures to Lorentzian profiles yields energies $E_{n}$ and widths $\Gamma_{n}$ of the ISRs. The width corresponds to the RET rate, i.e., it is inversely proportional to the lifetime: $\tau_{n}$ $=1 / \Gamma_{n}$.

We now turn to the discussion of the results. For low $n$, calculated energies of the ISRs coincide with early published data obtained with the same model potential (see Ref. 16 for detailed comparison with experiment). We shall only analyze the general trends obtained here thanks to the wide $n$ range of our study. We find that, similarly to the conventional ISs, the energies of the ISRs closely follow the behavior given by Eq. (1). Thus, with the quantum defect obtained from the linear fit to the $1 / \sqrt{\left|E_{n}\right|}$ quantity and listed in Table I, one can reproduce the calculated energies of the $n=1-6$ ISR series with precision better than $0.5 \%$. Note that a more negative quantum defect corresponds to larger binding energies of the ISR series.

Our main results concern the widths of the ISRs. We re- call that the widths of the ISRs are associated here with the rates of the energy-conserving one-electron RET into the metal. This is in contrast with the conventional ISs decaying via inelastic electron-electron scattering. Even though the efficiency of the one-electron transfer is usually assumed to be essentially higher than that of the multielectron effects, the latter can still contribute to the decay of the ISRs. We addressed this question performing two types of calculations: with and without inclusion of the optical potential $V_{0}(z)$ inside the metal. The resulting widths of the ISRs show no effect of the $V_{0}(z)$. This can be understood from the fact that the time during which an electron is trapped in front of the surface is determined by the finite reflectivity of the metalvacuum interface, i.e., by the RET. The many-body energy relaxation described with the $V_{0}$ potential is active once an electron is transferred into the metal. However, this electron is already lost from the point of view of the population of the quasistationary state. Therefore, many-body effects are responsible for the final states of transferred electrons, while the lifetime of the excited state is given by the RET rate. This result is quite different from the one obtained for the resonances induced within the projected band gap of the $\mathrm{Cu}(111)$ surface by alkali-metal $(A)$ adsorbates. ${ }^{18}$ In the $A / \mathrm{Cu}(111)$ system the RET is greatly suppressed, and both elastic and inelastic decay additively contribute to the width of the adsorbate-localized resonance.

Summary of the present results and their comparison with available experimental and theoretical data are presented in Table II. As a systematic trend, the width increases along the $\mathrm{Ag}(111), \mathrm{Cu}(111), \mathrm{Au}(111)$, and $\mathrm{Be}(0001)$ series, reflecting decrease of the energy of the top of the projected band gap with respect to the vacuum level. Indeed, ISRs are pinned to the vacuum level, so that going energetically further away from the projected band gap decreases surface reflectivity and increases the RET rate. Thus, the $n=1$ state at $\mathrm{Be}(0001)$ surface is basically $1 \mathrm{eV}$ broad, reflecting an extremely short lifetime $\tau \sim 0.7$ fs. For the given surface the lifetime of the ISRs increases with increasing $n$ (see discussion below). Observe that the $n=1$ states are within the projected band gap for $\mathrm{Ag}(111)$ and $\mathrm{Cu}(111)$ surfaces. Then, the large efficiency of the one-electron RET process is seen from the clear larger widths of the quasistationary $n=2$ ISR as compared to the calculated or measured many-body width of the $n=1$ IS. One can also compare the widths of the ISRs, $40 \mathrm{meV}(n=2)$ and $13 \mathrm{meV}(n=3)$ obtained here for $\operatorname{Ag}(111)$ and $\mathrm{Cu}(111)$ surfaces with linewidths of the order of $5 \mathrm{meV}(n=2)$ and $2 \mathrm{meV}(n=3)$ reported for the ISs lying in the projected band gap of $\mathrm{Ag}(100)$ and $\mathrm{Cu}(100) .^{28}$

For $\mathrm{Ag}(111)$ and $\mathrm{Cu}(111)$ surfaces calculated widths of the $n=2$ and 3 ISRs are in good agreement with TR2PPE experimental data. For $n=1$ and 2 ISRs of $\mathrm{Au}(111)$ our results are in agreement with an earlier theoretical study ${ }^{15}$ as well as with recent calculations performed with the same model potential as here. ${ }^{14}$ The experimental values obtained with two-photon photoemission for $n=1$ are overestimated by a factor of 2 . The origin of this discrepancy might be twofold. First, there is a clear experimental difficulty in measuring such short-lived states. ${ }^{25}$ Second, the one-dimensional model potential approximation might be too crude. However, 
TABLE II. Widths of the ISRs (meV). First column for each surface corresponds to the present results. Numbers in parentheses stand for the conventional IS stationary within the one-electron picture. $E_{u}$ is the energy of the top of the projected band gap with respect to the vacuum level. Th, theory; ex, experiment.

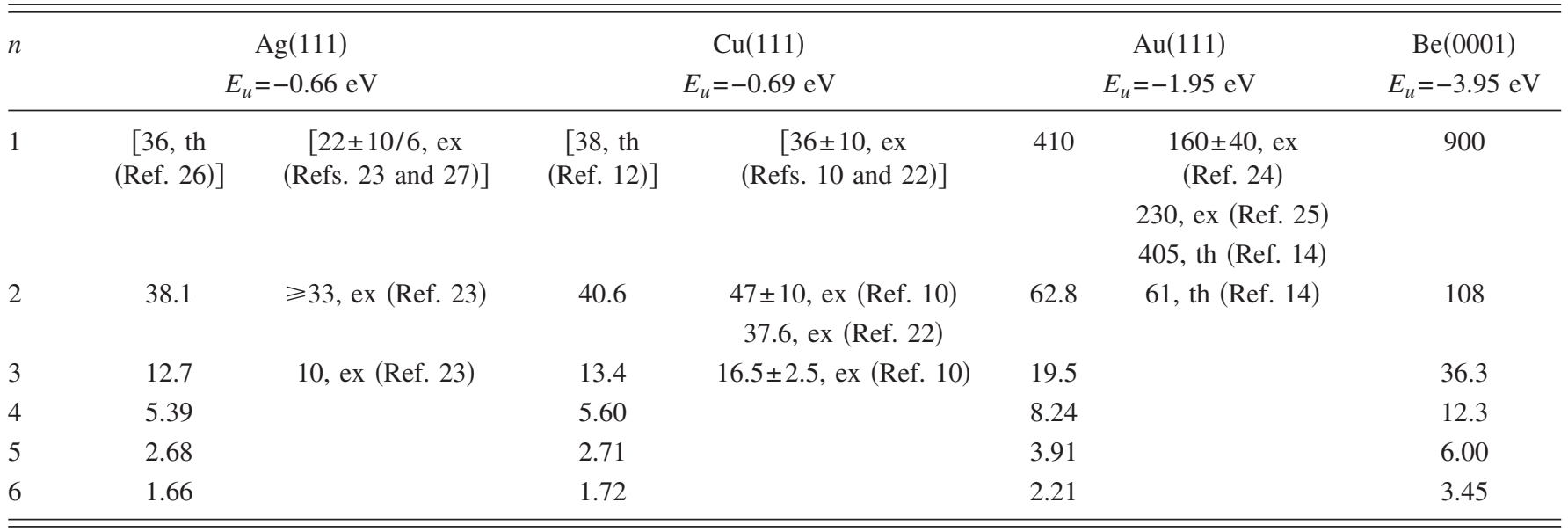

it is noteworthy that the same model potential does a perfect job for the lifetimes of the ISs and ISRs at many other surfaces. We believe that further experiments are needed for a definitive statement.

One remark is in order in connection with time-resolved studies. As follows from earlier data, the lifetimes of excited electrons in the bulk of $\mathrm{Cu}, \mathrm{Ag}$, and $\mathrm{Au}$ are in the 2-5 fs range (130-300 meV) for the ISR energies. ${ }^{29}$ Thus, for $\mathrm{Ag}(111)$ and $\mathrm{Cu}(111)$ the calculated ISR lifetimes are essentially larger than the corresponding bulk-electron lifetimes. Then the TR2PPE signal would indeed reflect the time during which an electron is trapped in front of the surface. On this time scale, as soon as an electron escapes through the surface barrier into the metal it is immediately lost because of the energy relaxation. The situation is quite different for, e.g., the $n=1$ ISRs of $\mathrm{Au}(111)$ and $\mathrm{Be}(0001)$. The RET is extremely fast in this case, much faster than the many-body relaxation in the bulk. Thus, an electron will basically instantaneously be transferred into the metal and the TR2PPE signal will reflect the excited electron lifetimes in the bulk as well as transport effects.

Figure 1 shows the principal quantum number $n$ dependence of the calculated widths of the image-state resonances. For the ISs an $n^{3}$ dependence of the many-body lifetimes has been predicted from penetration arguments. ${ }^{8}$ Experimental data $^{10,30}$ as well as $a b$ initio theoretical results ${ }^{12}$ show that this scaling law holds only approximately for $n \geqslant 2$, the main reason being the presence of extremely efficient decay channels into the lower image-state continua ${ }^{12}$ which are not accounted for by the simple penetration point of view. As for the ISRs, in Fig. 1 we observe almost perfect $n^{-3}$ scaling of the widths ( $n^{3}$ scaling of the lifetimes) through the entire series. This scaling can be understood from semiclassical arguments. Indeed, for an electron moving under the action of the image potential and colliding from time to time with the surface, the rate of the escape into the metal is given by the attempt (collision) frequency multiplied by the probability of the transmission through the surface. Since the difference in energies of the ISRs is relatively small, the transmission probability is basically constant through the $n$ series, while the collision frequency is proportional to $n^{-3} .31$ Therefore, theresulting RET rate (width) has an $n^{-3}$ dependence.

In summary, we have performed a detailed theoretical study of the binding energies, and particularly lifetimes, of the image-state resonances on clean metal surfaces. Systematic calculations performed within a wide $n$ range (1-6) for several metal surfaces, $\mathrm{Cu}(111), \operatorname{Ag}(111), \mathrm{Au}(111)$, and $\mathrm{Be}(0001)$, reproduce available experimental data and allow discussion of the dependence of the ISR lifetimes on the surface band structure. Similarly to the theoretical prediction for the many-body lifetimes of conventional ISs, we find $n^{3}$ scaling of the ISR lifetimes with the principal quantum number. This can be understood on the basis of semiclassical arguments connecting the probability of electron tunneling into the bulk metal with the period of its motion in the image potential. The present work establishes that the model potential successively applied to explain the properties of the ISs can be used as well for the ISRs. It thus opens the possibility for further theoretical studies for clean and adsorbate-coated surfaces. Finally, we believe that this work will stimulate new experimental studies.

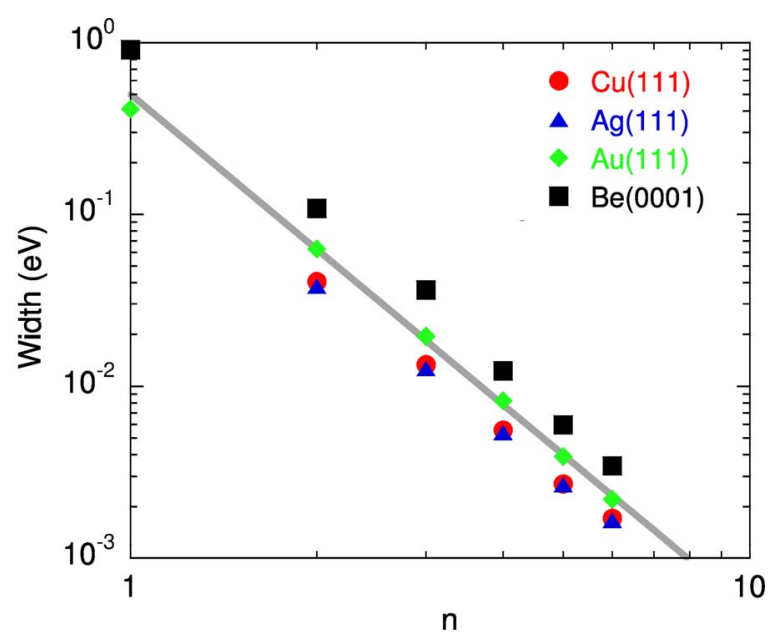

FIG. 1. (Color online) Calculated widths of the ISRs as functions of the principal quantum number $n$. Note the logarithmic scale used for both axes. Gray line traces the $0.5 n^{-3}$ function. 


\section{ACKNOWLEDGMENTS}

We acknowledge the support from the University of the Basque Country, the Basque Hezkuntza Saila, the Spanish
MCyT (Grant No. FIS2004-06490-C03-01), and the EC Sixth Framework Network of Excellence NANO-QUANTA (Grant No. NMP4-CT-2004-500198).
${ }^{1}$ X.-Y. Zhu, J. Phys. Chem. B 108, 8778 (2004).

${ }^{2}$ P. Avouris, R. E. Walkup, Annu. Rev. Phys. Chem. 40, 173 (1989).

${ }^{3}$ U. Höfer, I. L. Shumay, Ch. Reuß, U. Thomann, W. Wallauer, and Th. Fauster, Science 277, 1480 (1997).

${ }^{4}$ T. Hertel, E. Knoesel, M. Wolf, and G. Ertl, Phys. Rev. Lett. 76, 535 (1996)

${ }^{5}$ H. Petek, M. J. Weida, H. Nagano, and S. Ogawa, Science 288, 1402 (2000).

${ }^{6}$ N. V. Smith, Phys. Rev. B 32, 3549 (1985).

${ }^{7}$ M. Weinerlt, S. L. Hulbert, and P. D. Johnson, Phys. Rev. Lett. 55, 2055 (1985).

${ }^{8}$ P. M. Echenique and J. B. Pendry, J. Phys. C 11, 2065 (1978).

${ }^{9}$ P. M. Echenique and J. B. Pendry, Prog. Surf. Sci. 32, 111 (1989).

${ }^{10}$ M. Weinelt, J. Phys.: Condens. Matter 14, R1099 (2002).

${ }^{11}$ E. V. Chulkov, I. Sarría, V. M. Silkin, J. M. Pitarke, and P. M. Echenique, Phys. Rev. Lett. 80, 4947 (1998).

${ }^{12}$ P. M. Echenique, J. M. Pitarke, E. V. Chulkov, and A. Rubio, Chem. Phys. 251, 1 (2000).

${ }^{13}$ S. Crampin, Phys. Rev. Lett. 95, 046801 (2005).

${ }^{14}$ C. D. Lindstrom, D. Quinn, and X.-Y. Zhu, J. Chem. Phys. 122, 124714 (2005).

${ }^{15}$ L. Jurczyszyn, Surf. Sci. 247, 158 (1991); 259, 65 (1991).

${ }^{16}$ E. V. Chulkov, V. M. Silkin, and P. M. Echenique, Surf. Sci. 437, 330 (1999).

${ }^{17}$ S. Yang, R. A. Bartynski, G. P. Kochanski, S. Papadia, T. Fondén, and M. Persson, Phys. Rev. Lett. 70, 849 (1993).

${ }^{18}$ A. G. Borisov, J. P. Gauyacq, E. V. Chulkov, V. M. Silkin, and P. M. Echenique, Phys. Rev. B 65, 235434 (2002).
${ }^{19}$ R. Kosloff, J. Phys. Chem. 92, 2087 (1988).

${ }^{20}$ D. Neuhauser and M. Baer, J. Chem. Phys. 91, 4651 (1989).

${ }^{21}$ M. A. Van Hove, W. H. Weinberg, and C. M. Chan, Low Energy Electron Diffraction (Springer-Verlag, Berlin, 1986).

${ }^{22}$ M. Wolf, E. Knoesel, and T. Hertel, Phys. Rev. B 54, R5295 (1996).

${ }^{23}$ C. B. Harris, N. H. Ge, R. L. Lingle, J. D. McNeill, and C. M. Wong, Annu. Rev. Phys. Chem. 48, 711 (1997).

${ }^{24}$ Th. Fauster and W. Steinmann, in Photonic Probes of Surfaces, edited by P. Halevi, Electromagnetic Waves: Recent Developments in Research, Vol. 2 (North-Holland, Amsterdam, 1995), Chap. 8, p. 347.

${ }^{25}$ C. Reuß, W. Wallauer, and Th. Fauster, Surf. Rev. Lett. 3, 1547 (1996).

${ }^{26}$ A. García-Lekue, J. M. Pitarke, E. V. Chulkov, A. Liebsch, and P. M. Echenique, Phys. Rev. B 68, 045103 (2003).

${ }^{27}$ J. D. McNeill, R. L. Lingle, Jr., N.-H. Ge, C. M. Wong, R. E. Jordan, and C. B. Harris, Phys. Rev. Lett. 79, 4645 (1997).

${ }^{28}$ I. L. Shumay, U. Höfer, Ch. Reuß, U. Thomann, W. Wallauer, and Th. Fauster, Phys. Rev. B 58, 13974 (1998).

${ }^{29}$ V. P. Zhukov, F. Aryasetiawan, E. V. Chulkov, I. G. de Gurtubay, and P. M. Echenique, Phys. Rev. B 64, 195122 (2001).

${ }^{30}$ W. Berthold, F. Rebentrost, P. Feulner, and U. Höfer, Appl. Phys. A: Mater. Sci. Process. 78, 131 (2004).

${ }^{31}$ The period of classical motion in the $-1 / 4 z$ potential can be straightforwardly obtained following L. D. Landau and E. M. Lifshitz, Mechanics, Course of Theoretical Physics, Vol. 1 (Butterworth-Heinemann, Oxford, 1999), Chap. 3, Sec. 11, problem 2. 